\title{
Multipoint temperature measurements in gas flows using 1-D laser-induced grating scattering
}

\author{
Christopher Willman ${ }^{1} \cdot$ Paul Ewart $^{1}$ (D)
}

Received: 30 September 2016 / Revised: 7 November 2016 / Accepted: 12 November 2016 / Published online: 22 November 2016 (C) The Author(s) 2016. This article is published with open access at Springerlink.com

\begin{abstract}
A technique is reported for simultaneous, timeand space-resolved measurements of temperature using laser-induced thermal grating scattering, LITGS, from four points on a 1-D line. Signals from four separate points on the line, separated by $1 \mathrm{~mm}$, in toluene-seeded nitrogen flows, were imaged onto a fibre-optic array and delivered to separate photodiode detectors to record their temporal evolution from which the temperatures at each point were derived with a spatial resolution of $1 \mathrm{~mm}$ and a precision of $0.7 \%$ at atmospheric pressure. Effects of variation of composition on the accuracy of the measurements were compensated by a calibration method providing good agreement with values inferred from thermocouple measurements. Temperature gradients at the boundary between parallel gas flows and at the surface of hot and cold surfaces were measured with a resolution of $5 \mathrm{~K} \mathrm{~mm}^{-1}$. Extension of the technique to more measurement points and improvements in spatial resolution are briefly discussed.
\end{abstract}

\section{Introduction}

The temperature of combusting and non-combusting flows is an important parameter in a wide range of problems including heat transfer to surfaces in internal combustion engines, cooling of turbine blades in aero-engines and studies of thermal boundary layers in hypersonics and the physics of spacecraft entry or re-entry into atmospheres. (Lucht 1991; Kays and Crawford 1993; Heppenheimer 2006) Of

Paul Ewart

p.ewart@physics.ox.ac.uk

1 Clarendon Laboratory, Department of Physics, University of Oxford, Parks Road, Oxford OX1 3PU, UK particular concern is the measurement of temperature gradients close to surfaces exposed to hot or cold gas flows. Accuracy and precision of the measurement are also important together with high spatial and, in some circumstances, high temporal resolution. Since physical probes are both invasive and perturbing, attention has been centred on optical methods which are inherently non-invasive. Twodimensional imaging techniques using linear optical processes, such as Rayleigh scattering or planar laser-induced fluorescence, PLIF, have been applied to both combusting and non-combusting flows (Eckbreth 1996; Kohse-Höinghaus and Jeffries 2002; Schulz and Sick 2005). Such methods, however, suffer from a number of limitations such as low signal levels and interference from particulate matter in the case of Rayleigh scattering and calibration issues with PLIF methods. Nonlinear optical techniques, including coherent anti-Stokes Raman scattering, CARS, degenerate four-wave mixing, DFWM, and polarization spectroscopy, PS, have been demonstrated for both 2-D and 1-D (i.e. line) imaging of both temperature and concentrations (Dreier and Ewart 2002; Kiefer and Ewart 2011). Most recently, impressive performance of 1-D and 2-D imaging of temperature and concentrations has been demonstrated using fs-CARS (Bohlin and Kliewer 2013, 2014; Bohlin et al. 2013). These techniques, however, tend to rely on highpower lasers and involve complex optical arrangements and non-trivial data analysis.

Another nonlinear optical technique, laser-induced grating scattering, LIGS, has been developed over the past two decades and demonstrated for thermometry in both combusting and non-combusting gases including in-cylinder measurements in an internal combustion engine (Eichler et al. 1986; Cummings 1994; Paul et al. 1995; Latzel et al. 1998; Williams et al. 2014). Almost all of these applications of LIGS have involved "point-like" measurements where the 
measurement volume is defined by the size of the overlap region of the two pump beams and that of the interrogating probe beam. Extension of the method to measurements in 2-D across a plane would require simultaneous recording of the time behaviour of the LIGS signal from many points in the plane and so, at present, is not technically feasible. Two-dimensional imaging using LIGS has been reported but using only the time-integrated intensity of the signal (Hemmerling and Stampanoni-Panariello 1993). In a recent study, LIGS was used to provide an in situ calibration measurement for 2-D thermometry based on two-colour PLIF (Ewart et al. 2015). Extension of LIGS temperature measurements from a point to a 1-D line was first demonstrated by using a streak camera to record the temporal behaviour from LIGS signals generated along a line (Stevens and Ewart 2006). Sheet-like pump beams were used to define the line by the intersection of the two sheets and probe light scattered from the line was imaged onto the input slit of a streak camera. The streak camera image thus provided a spatially resolved LIGS signal, showing the temporal oscillations in signal intensity at each point on the line, from which the temperature was derived with an uncertainty of $0.3 \%$ and a spatial resolution of $150 \mu \mathrm{m}$ along the line. These measurements were taken in a cell at uniform temperature, and so no temperature gradient was probed.

Although this previous work demonstrated the principle of 1-D LIGS thermometry, the full potential of the technique was not realized owing to limitations imposed by the performance of the streak camera. The time interval covered by the streak sweep was only $120 \mathrm{~ns}$, whereas the LIGS signals from a target gas had durations up to $1 \mu \mathrm{s}$. As a result, the camera could record only part of the signal and so the precision of the single-shot, time-resolved measurement was limited. The precision of $0.3 \%$ was achieved only by "stitching together" a sequence of LIGS signals recorded at increasing delay times to cover the entire signal duration. This limitation is inherent in the design of most streak cameras. The problem arises from the need to have both a detector rise time of the order of $1 \mathrm{~ns}$ and a swept record of between 500-ns and 1- $\mu$ s duration. (The oscillation frequencies of LIGS signals are typically in the range 10-100 MHz.) The ratio of the time sweep to rise time required is therefore of the order of 500. Commercially available streak cameras can provide a ratio of up to 200 which is insufficient to give the required temporal resolution over the relatively long signal times encountered with LIGS at pressures of 1 bar and higher. In any case, the expense of streak cameras would put them beyond the budget of many potential users of the technique.

In the present paper, we report an alternative scheme which replaces the expensive, and time-limited, streak camera with a much less expensive system based on fast photodiodes. This system, apart from being of much lower cost, overcomes the limitation of the sweep-time/rise-time ratio of the streak camera at the expense of measuring at a few discrete points rather than continuously along the line. In what follows, we give, firstly, a brief description of the underlying physics of LIGS to explain how the temperature is derived from the time-resolved recording of the signal. Secondly, we outline the experimental apparatus and procedure used to generate and record the spatially resolved LIGS signal including consideration of effects that determine the absolute accuracy and precision of temperatures derived from the measurements. Demonstrations of the technique are then described for two particular cases; firstly, a temperature gradient established in a flow consisting of two streams of gas at different temperature, and secondly, gas flows over a surface where the gas and surface are at different temperatures. Finally, some future applications of the technique and extensions to more measurement points are discussed.

\section{Thermometry using LIGS}

The basic principles of LIGS have been reviewed recently with details of the underlying physics being extensively reported in the literature, and so only a brief outline is provided here for convenience (Kiefer and Ewart 2011). A grating-like structure is induced in the gaseous medium by the interference of two, short-duration laser pulses (typically a few ns) - the pump pulses, derived from the output of a single pulsed laser. Following absorption of energy by, and subsequent collisional relaxation of, some molecular species, a grating-like perturbation of temperature and density is established in the gas. The spatial period of this grating structure, $\Lambda$, is determined by the wavelength of the light and the crossing angle. Associated with the generation of this stationary "thermal grating", a standing acoustic wave is launched which modulates the scattering efficiency of the grating at a frequency, $f_{\text {osc }}$, determined by $\Lambda$ and the speed of sound $c_{s}$. This specific process is designated laserinduced thermal grating scattering, LITGS, to distinguish it from a more general LIGS process that can include a non-resonantly generated grating arising from electrostriction. Light scattered from a c.w. probe laser incident on the induced grating at the Bragg angle is modulated as the phase of the acoustic wave evolves leading to periodic cancellation of the stationary grating. The scattered signal can be detected and the frequency of the periodic modulation measured with high precision. The speed of sound derived from this measured frequency allows the temperature to be calculated from the relation:

$$
T=\frac{m}{\gamma} \frac{\Lambda^{2}}{k_{\mathrm{B}}} f_{\mathrm{osc}}^{2}
$$


where $m$ is the mean molecular mass of the gas, $\gamma$ is the ratio of specific heats at constant pressure and volume and $k_{\mathrm{B}}$ is Boltzmann's constant.

This relationship shows that the composition of the gas needs to be known with sufficient accuracy to determine the ratio of $m / \gamma$, an issue that will be considered in the analysis of experimental data.

\section{Experimental apparatus and procedure}

The optical arrangement for generating and detecting the LITGS signal is shown schematically in Fig. 1. The pump pulses were derived from the frequency quadrupled output of a Nd:YAG laser (Continuum 8000) at $266 \mathrm{~nm}$ having a duration of about $5 \mathrm{~ns}$ and operating at $10 \mathrm{~Hz}$ repetition rate. After splitting the original beam, and compensating for the optical delay involved, the two pulses, containing $4 \mathrm{~mJ}$ energy per beam, were caused to intersect at a small angle $\theta$ in the interaction region. By adjusting $\theta$, a suitable grating period $\Lambda$ was established and its value in the present work $\Lambda$ was $6.6 \pm 0.3 \mu \mathrm{m}$. A c.w. probe beam from a diode-pumped solid-state laser (Laser Quantum, Ventus) with power of $750 \mathrm{~mW}$ at a wavelength of $660 \mathrm{~nm}$ was arranged to intersect the induced grating at the Bragg angle using a system of apertures in a suitably designed mask to facilitate alignment. Similarly, a guide beam, derived from the same laser, was aligned and used to indicate the direction of the generated signal. Both pump and probe beams were tailored using cylindrical lens optics to form intersecting sheets $1 \mathrm{~mm}$ thick and $6 \mathrm{~mm}$ high. The scattered signal was imaged using a lens system giving 1:1 relay of the intensity distribution along the interaction line to the image plane. A linear array of four fibre optics (core diameter $1.0 \mathrm{~mm}$, cladding $35 \mu \mathrm{m}$ ) was placed in the image plane so that signal from four points in the interaction region was collected with each signal directed to a separate fibre. Each individual signal was then conveyed by the respective fibre to a separate photodiode (Thorlabs DET10A/M) and the resulting signals recorded using a 4-channel digital oscilloscope (LeCroy Waverunner 625Zi). In this way, the LITGS signals from four points, separated by $1.07 \mathrm{~mm}$ on the line, were simultaneously recorded with the $2.5 \mathrm{GHz}$ bandwidth of the scope over the entire signal duration of up to $500 \mathrm{~ns}$. The sample rate of $20 \mathrm{GS} / \mathrm{s}$ is more than adequate to determine the waveform of the oscillating LITGS signal. The spatial separation of the points on the line in the interaction region being probed in this arrangement, using 1:1 imaging, matches that of the fibre optics in the image plane. It is a simple matter to probe points at different separations by using an image system with a magnification greater or less than unity - the only limitation being the requirement to ensure adequate signal intensity reaches each photodiode if the image is magnified.

Experiments were conducted to measure temperatures in two different flow situations. Firstly, a "hot/cold flow" consisting of two adjacent and parallel flows at different temperatures to provide a stable temperature gradient at the boundary between the flows. Secondly, flows at a uniform temperature were arranged to impinge tangentially on a stationary surface at a temperature either higher or lower than that of the gas flow. The temperature gradient in this case was established by the difference between the temperature of the flow and that of the surface-a situation commonly encountered in boundary layer problems.

The flows were seeded with toluene by passing the nitrogen flow over a liquid toluene reservoir placed on a digital balance to measure the rate of mass loss and hence give a measure of the toluene concentration in the flow. The toluene concentration was set to typically $2.3 \%$ which provided adequately strong LITGS signals.
Fig. 1 Optical arrangement for generating LITGS signals along a line. The line is defined by the intersection of the two pump beams formed into sheets. The signals from each point on the line are imaged to form a line image such that the 4-optical fibre array in the image plane selects the signals from four separate points on the line which are then detected on separate photodetectors

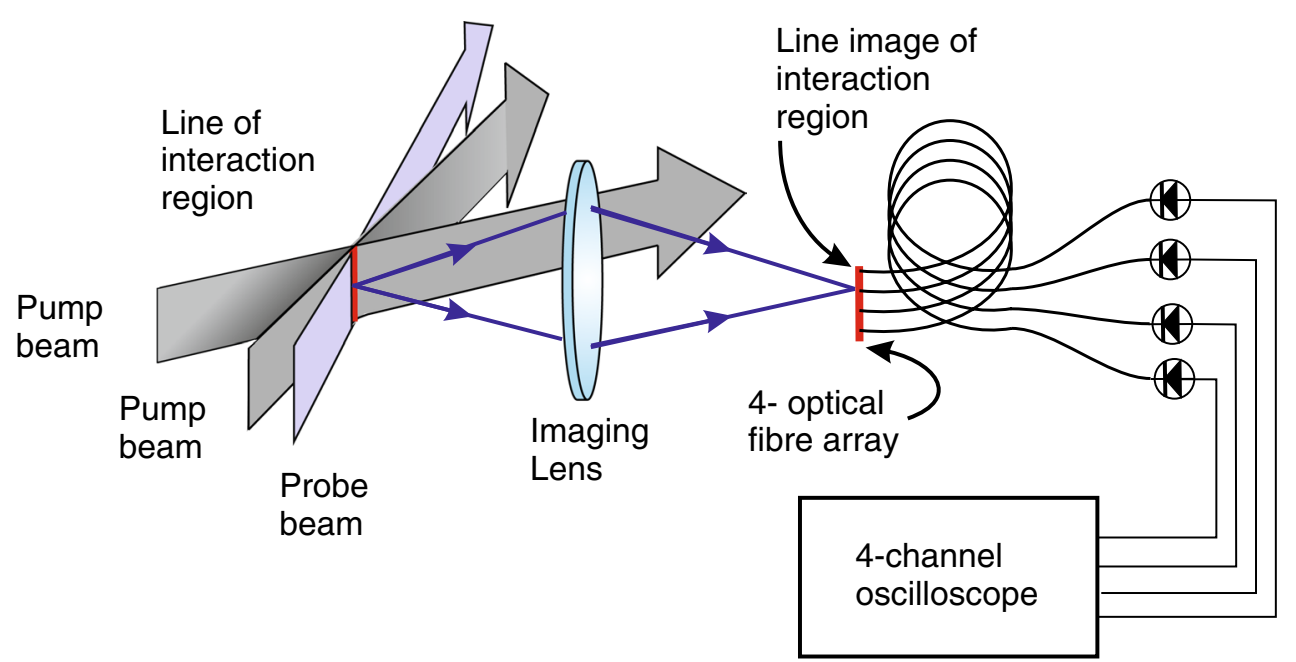




\subsection{Calibration and perturbation issues}

Two practical difficulties were encountered that required care to ensure accuracy of the temperature values derived from the measurements. Firstly, in the case of the hot/cold flow air entrainment at the edge of the flows led to unevenness in the toluene density and thus a difference in signal intensity from the hot and cold flows towards the edges of the flows. Although the derivation of temperature does not rely on an intensity measurement, the difference indicated a difference in toluene density and hence in the value of $m / \gamma$ which can potentially affect the value of the derived temperature. Secondly, the need to concentrate the pump energy into a line led to high intensities at some parts of the interaction region owing to beam inhomogeneity (laser "hot spots"). When higher pump intensities were used, the localized heating in such spots perturbed the temperature by a measurable amount. This effect was readily detected by variation in the derived temperature in situations where the temperature was known to be uniform, e.g. in roomtemperature flows. As in previous work, the intensity of the pump was therefore reduced and kept to a minimum in order to avoid such perturbations.

There are therefore two separate effects that result in independent sources of error in the temperatures derived from the LITGS signals: (a) variation of $m / \gamma$ across the measurement region resulting from differences in toluene concentration and (b) differential heating in the measurement region as a result of absorption from the pump beams, especially in local "hot spots". To complicate matters further, these two effects are not, in general, independent since the amount of local heating by the pump lasers depends on the absorption coefficient of the gas which varies with absorber concentration. In practice, it is usually possible to minimize pump-heating effects by using the minimum power required to achieve a measurable LITGS signal.

In principle, both sources of error can be reduced or eliminated by using very small concentrations of absorber such that the value of $m / \gamma$ does not vary appreciably from that of the nitrogen flow or entrained air and the amount of energy absorbed in the measurement volume is also sufficiently small to result in negligible change in the local temperature. Errors associated with uncertainty in, or variation of, the gas composition have been discussed in previous work (Williams et al. 2014). The effect of variation in concentration of the toluene absorber from an assumed value is shown in Fig. 2 for reference concentrations between 0 and $2.3 \%$. This figure shows that for toluene concentrations below $0.5 \%$ variations of up to $100 \%$ of this value will result in errors of less than $1 \%$. (Note that, in this figure, the colour scale representing the error in the derived temperature is capped at $2.5 \%$ to display more visibly the sensitivity to variations in concentration giving errors of

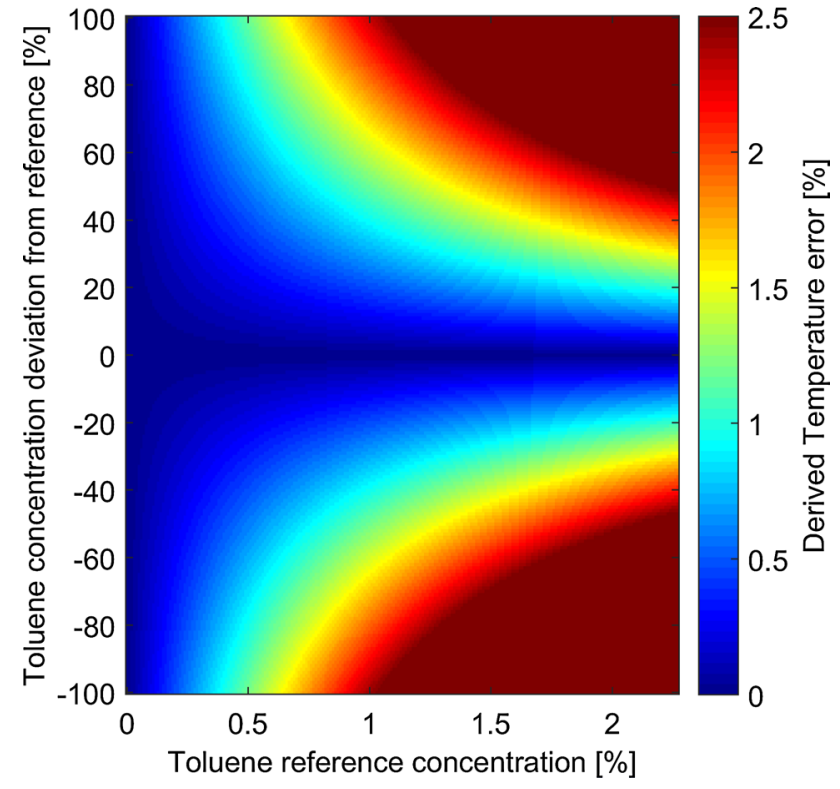

Fig. 2 Plot showing percentage error in derived temperature for deviations from the reference concentration of toluene plotted on the $x$-axis. The plot illustrates that higher toluene concentrations lead to larger errors for a given percentage deviation in concentration. To limit errors to $\leq 1 \%$ requires use of concentrations less than $0.5 \%$

the order of $1 \%$. Errors above $2.5 \%$ are not differentiated in this plot.)

In practice, however, in these experiments designed to demonstrate the principle of multi-point thermometry using LITGS, the degree of toluene seeding was increased to $2.3 \%$ to ensure strong and easily measurable signals for all the data presented here. As a result, it was necessary, in the present work, to make some calibration measurements to account for variations in the composition of the gas and to ensure that local heating was kept to a minimum. Fortunately, it was found that compositional effects were consistent and reproducible allowing calibration measurements to be made that reduced the associated errors significantly.

Calibration was achieved using a flow of toluene-seeded nitrogen at room temperature injected into room-temperature static air and measurements taken at each of the four measurement points defined by the arrangement described above. Since the temperature is assumed to be uniform under these conditions, any variation in derived temperature can be assigned to effects of spatial variation of the composition. A calibration constant could thus be derived for each measurement position defined by the imaging system on each of the four fibre-optic/detector systems. This constant defined the proportionality constant required to bring the derived temperature at each point into agreement with the room-temperature value.

In addition to its effect on $\mathrm{m} / \gamma$, the variation in composition is also associated with variation in the amount of 
energy absorbed and hence of local heating by the pump beams. The calibration procedure then also accounts for any slight perturbation of the local temperature caused by heating by the pump beams. Estimates based on the absorption coefficient and incident energy in the pump pulses suggest a maximum temperature increase in approximately $20 \mathrm{~K}$ in approximate agreement with the value inferred from calibration measurements extrapolated to zero toluene composition. It will be straightforward to reduce this temperature perturbation to a value lower than the measurement precision by one or more of the following procedures. Firstly, by operating with the minimum concentration of toluene needed to produce an acceptable $\mathrm{S} / \mathrm{N}$ ratio, the degree of heating will also be lowered from its present value in direct proportion to the reduction in concentration and hence absorption. Secondly, the energy per pulse in the pump beams can be reduced to the level where the derived temperature is independent of the pulse energy. Most effective of all will be the replacement of the photodiodes, which require relatively high signal intensity for measurable signals, by photomultipliers. These devices, giving orders of magnitude increase in sensitivity, will allow the use of both lower concentration of toluene, or other absorber, and lower pulse energies in the pump beams. The pump energy and concentration levels used in the present work were chosen to give reliable and high $\mathrm{S} / \mathrm{N}$ levels to demonstrate the principle of the technique. In practice, use of lower concentrations $\sim 1 \%$ or less, and lower pump energy will certainly be feasible using photomultipliers instead of photodiodes. By these means, the induced temperature change by beam heating will be reduced to a value less than the measurement precision of $\sim 1 \%$. In the present work, the effects of local heating by hot spots in the pump beams was minimized by using pump energies as low as possible consistent with measurable signal intensities and checking that the derived temperatures did not vary with increases in laser intensity.

Calibration of the measurement system using a roomtemperature flow, however, does not guarantee that changes in the flow conditions with heated flows will be compensated in exactly the same degree. However, for the purposes of this demonstration experiment, such secondary compositional variations are assumed to be of less significance since the temperature ranges investigated did not exceed $70 \mathrm{~K}$ above room temperature.

\subsection{Temperature gradient in a flow}

Experiments to measure a temperature gradient were conducted using a flow consisting of two parallel and adjacent horizontal flows of nitrogen seeded with toluene. This system provides a dynamically stable temperature distribution that avoids complications from convection currents in a static gas with a temperature gradient. The flow was established by feeding two separate flows to a nozzle through two parallel pipes which terminated in a diffuser at the nozzle entrance. The nozzle exit consisted of a $4 \times 8 \mathrm{~mm}$ slot (internal dimensions) with a $150-\mu \mathrm{m}$ stainless steel wall separating the two $4 \mathrm{~mm}$ diameter flows. The flow rates were adjusted to give a rate of $1.2 \mathrm{~L} / \mathrm{min}$ in each flow. This provided an approximately laminar flow (Reynolds number 140) to minimize turbulent mixing of the two parallel flows.

Each of the flows was independently seeded with toluene, and one was conducted through a resistively heated tube, whilst the other flow remained at room temperature. The heated flow temperature was monitored using a thermocouple and adjusted in the range 292-357 K. The heated flow was directed to the upper side of the divided horizontal nozzle in order to minimize buoyancy effects that would arise if the hotter flow was below the colder one. The combined flow was positioned to pass through the measurement line formed by the intersecting pump sheets as described above. The nozzle was mounted on a translation stage to facilitate positioning the measurement plane at different distances from the exit.

\subsection{Flow over surface}

A single-orifice nozzle of diameter $8 \mathrm{~mm}$ was shaped and oriented to produce a laminar flow across a horizontal metal surface. The flow again consisted of $\mathrm{N}_{2}$ gas seeded with $2.3 \%$ toluene vapour as described above. Two cases were studied (a) room-temperature flow over a heated surface and (b) heated flow over a cold surface. The surfaces studied were of aluminium plates of thickness $18 \mathrm{~mm}$ and diameter $87 \mathrm{~mm}$. In case (b), the toluene-seeded $\mathrm{N}_{2}$ flow was passed through a resistively heated tube to raise the gas temperature in the range $290-350 \mathrm{~K}$. The metal surface was cooled by a flow of mains water at $278 \mathrm{~K}$ through pipes to the underside of the metal plate. In case (a), the metal plate was heated by resistive heaters attached to the underside of the plate. In both cases, the surface temperature was measured using type $\mathrm{K}$ thermocouples placed in contact with the surface exposed to the flow. The metal plates were mounted on a vertical translation stage to allow the interaction region - the vertical line defined by the intersecting pump beams - to be positioned such that the lowest measurement point was located $500 \mu \mathrm{m}$ above the surface. Thus, signals were collected effectively from points on a line normal to the surfaces at distances of approximately $0.5,1.5,2.5$ and $3.5 \mathrm{~mm}$.

Prior to each data collection run, the system was calibrated by using a flow of toluene-seeded $\mathrm{N}_{2}$ at room 
temperature as described above. Variations in the toluene concentration across the flows and the associated variation in $m / \gamma$ were thus characterized and the calibration constant determined for each measurement position along the line.

\section{Results and analysis}

Figure 3 shows typical single-shot LITGS signals recorded on each of the four detector channels in a simple flow of toluene-seeded $\mathrm{N}_{2}$ where the measurement points are selected from a line perpendicular to the flow. The variation in signal strength is accounted for by the difference in absorber (toluene) concentrations at the edges of the flow as a result of dilution by entrained air and also lower intensity of the pump beams at the edges of the beams.

These data show the typically high signal-to-noise ratio of the single-shot LITGS signals from which the temperature can be derived at each point on the line. Since the flow systems investigated were dynamically stable, it was possible to improve this further by averaging over 200 single shots. In the data presented in the following sections, the data points indicate the mean and the error bars indicate the standard deviation over 200 single shots after correction for compositional effects.
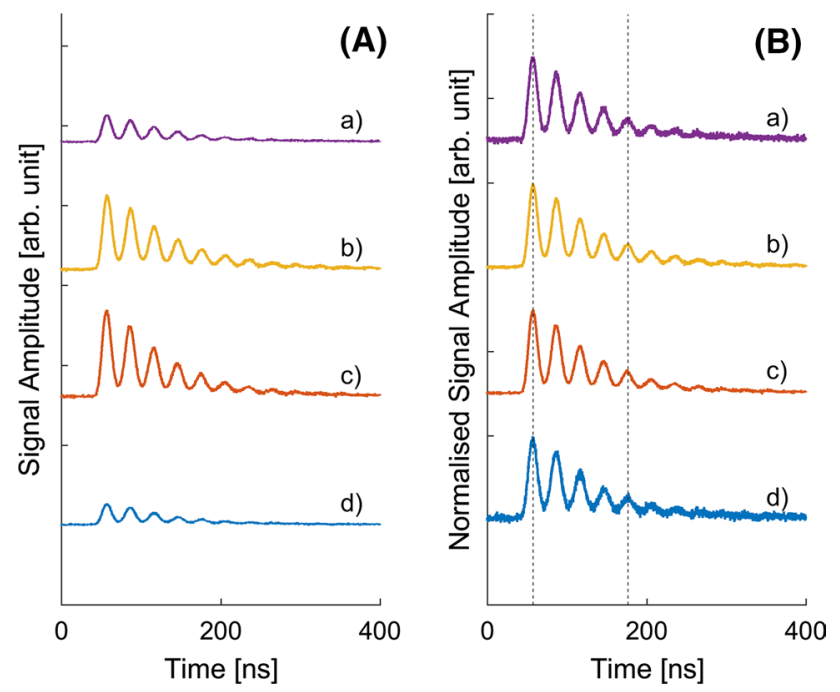

Fig. 3 Simultaneously recorded single-shot LITGS signals from points $(a),(b),(c)$ and $(d)$ on a 1-D line separated by approximately $1 \mathrm{~mm}$ in a uniform temperature flow at room temperature. A Raw signals on each photodetector; the variation in signal intensity from (a) top to (d) bottom arises largely from slight differences in toluene concentration and laser intensity in the pump sheets (see text). B The same data as shown in (A) normalized to the same signal level to show the same frequency of oscillation at each point under calibration conditions of uniform temperature. Two vertical lines (dashed) are shown as a guide to the eye. The zero time point corresponds to the time at which the oscilloscope was triggered

\subsection{Hot and cold flow}

The interaction region formed by the intersecting pump sheets was a vertical line of height $6 \mathrm{~mm}$ positioned at a right angle to the horizontal flows and $3 \mathrm{~mm}$ from the nozzle exit. The centre of the line was aligned with the horizontal boundary between the two flows. When imaged on to the fibre-optic array, using 1:1 imaging, this resulted in the signals being detected from positions 0.5 and $1.5 \mathrm{~mm}$ above and below the boundary layer between the flows. The axes of the flows were at $2 \mathrm{~mm}$ above and below the boundary, and so the top and bottom measurement points were approximately $0.5 \mathrm{~mm}$ from the centre line of each flow. The temperatures derived from the LITGS data recorded at each of the four measurement points are shown in Fig. 4.

The lower set of data was obtained with both flows at room temperature, $293 \mathrm{~K}$, and provided the calibration measurements to account for any variation in toluene concentration arising from different densities in heated flows or mixing with room air-thus, the temperature at each of these points is fixed at $293 \mathrm{~K}$. The error bars indicating the measurement precision of $0.7 \%$ are determined by the standard deviation of the values derived from 200 laser shots, i.e. in a $20 \mathrm{~s}$ measurement time at $10 \mathrm{~Hz}$. The upper two data sets represent the cases where the upper flow was

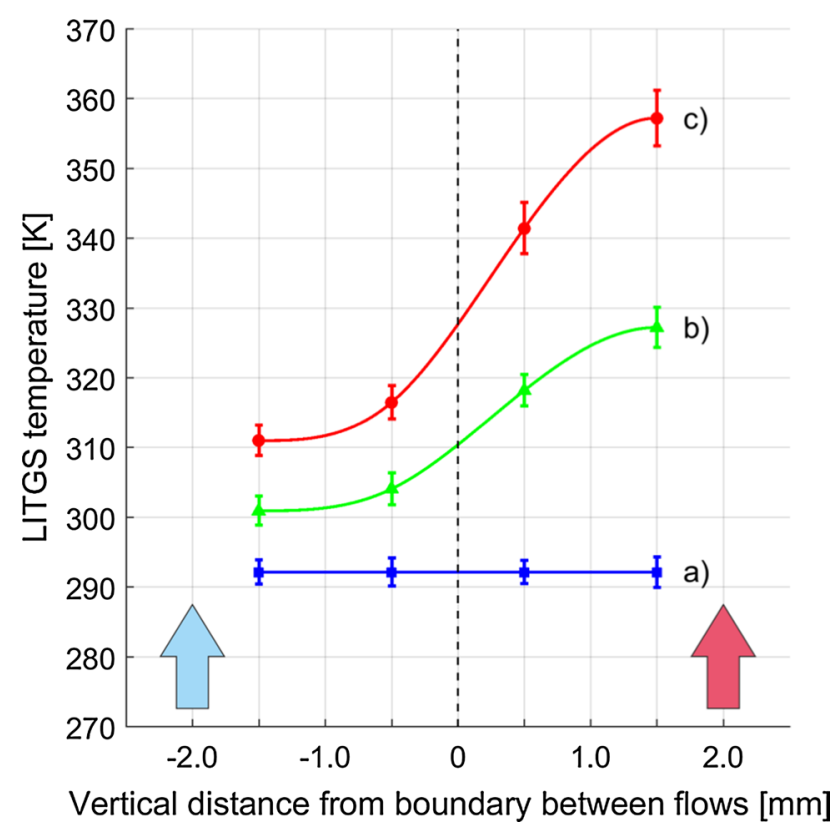

Fig. 4 Simultaneous measurement of temperature across a horizontal dual-temperature flow (see text). The location of the boundary between the two flows is indicated by the dotted line. The axes of the "hot" and "cold" flows are indicated by the arrows at +2.0 and -2.0 , respectively. $a$ Data for both flows at ambient temperature when corrected for composition and beam intensity effects to yield "room temperature" of $293 \mathrm{~K}, b$ and $c$ data from cases with upper flow heated at two different levels of power input to heater 
heated to successively higher temperature by increasing the current to the resistive heater in the upper flow tube. The lines through the data points are cubic splines added as a guide to the eye and illustrate the good spatial resolution achieved. With the current imaging geometry, this uncertainty sets a lower limit of $5 \mathrm{~K} \mathrm{~mm}^{-1}$ for the resolution of the temperature gradient.

\subsection{Hot and cold flows over surfaces}

Figure 5 shows temperature measurements obtained for a flow of toluene-seeded $\mathrm{N}_{2}$ at room-temperature flowing across a plate subjected to differing amounts of heating. The lowest trace (a) shows the data for an unheated plate at room temperature similar to the data acquired to compensate for variation in composition. The error bars, again specified by the standard deviation of 200 single shots, indicate the high precision of the measurements.

Four further sets of data are shown for increasing degree of heating applied to the plate resulting in the temperatures recorded by the surface contact thermocouples of 304, 318, 340 and $381 \mathrm{~K}$. In each case, the measurements in the flow show the expected steepening of the temperature gradient closer to the surface. Extrapolation of the cubic spline fits to the data points to the surface yield surface temperatures

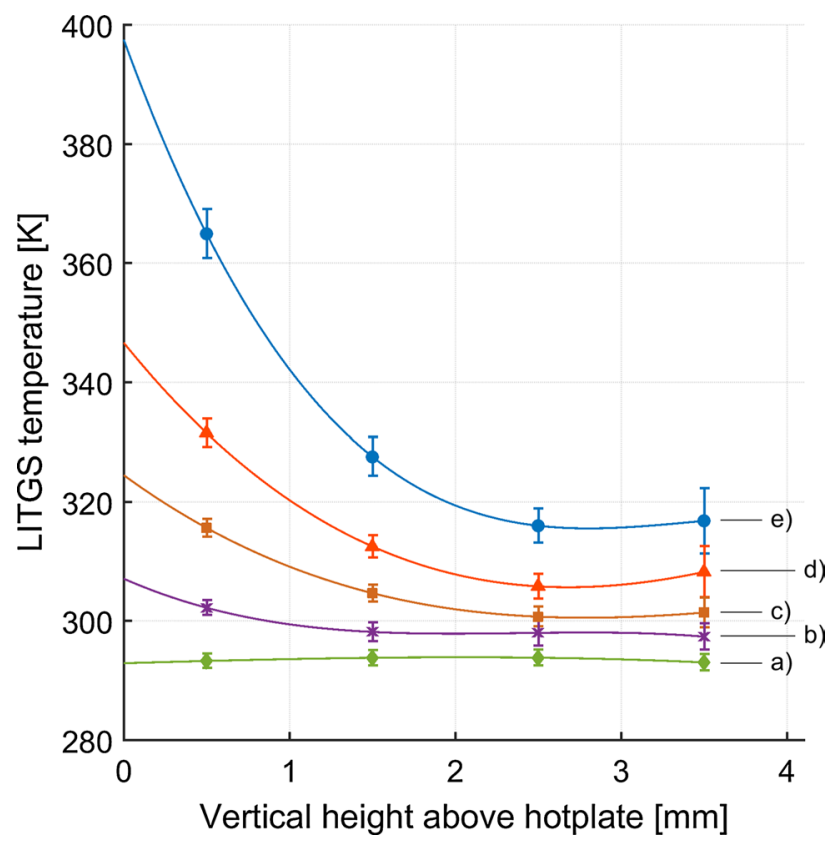

Fig. 5 Simultaneous measurements along a vertical line above a horizontal heated plate. Data sets are shown for different temperatures of the plate surface, $T_{\mathrm{P}}$ as measured by a thermocouple: a $293 \mathrm{~K}$, b $304 \mathrm{~K}$, c $318 \mathrm{~K}$, d $340 \mathrm{~K}$ and e $381 \mathrm{~K}$. The lower data set obtained for the plate at room temperature is similar to that which provided the calibration for variation in toluene concentration (see text). The curves are fitted splines as a guide to the eye somewhat higher than the values indicated by the thermocouples. This may again suggest that the thermocouple values are systematically decreased by less than perfect thermal contact with the surface or cooling effect of the flow across the wire near the junction.

Some heating of the flow is apparent at the higher plate temperatures as a result of conductive or radiative heating. We note also the slight increase in the standard deviation of the measurements at the higher-temperature ranges both at the surface and at the upper point $3.5 \mathrm{~mm}$ from the surface. This may be due to effects of turbulence and entrained air from the cooler room air outside the flow but also because the signals at these positions are weaker as a result of lower laser intensity and lower toluene concentration as a result of the mixing.

The data obtained in case (b), the heated flow impinging tangentially on a cold plate, are shown in Fig. 6. As before, the data for the room-temperature flow and plate at the same temperature provided the data used to calibrate the measurements for any variation in $m / \gamma$ across the flow by assuming the temperature at each of these points was the same as ambient temperature, $293 \mathrm{~K}$. When the cooling water was applied to the plate, the surface temperature dropped below room temperature as shown in the figure. The third flow condition where the flow was heated to $309 \mathrm{~K}$, as measured by a thermocouple at the nozzle exit, shows the cooling effect of the plate at the position $0.5 \mathrm{~mm}$ from the surface. The temperature at the furthest point from

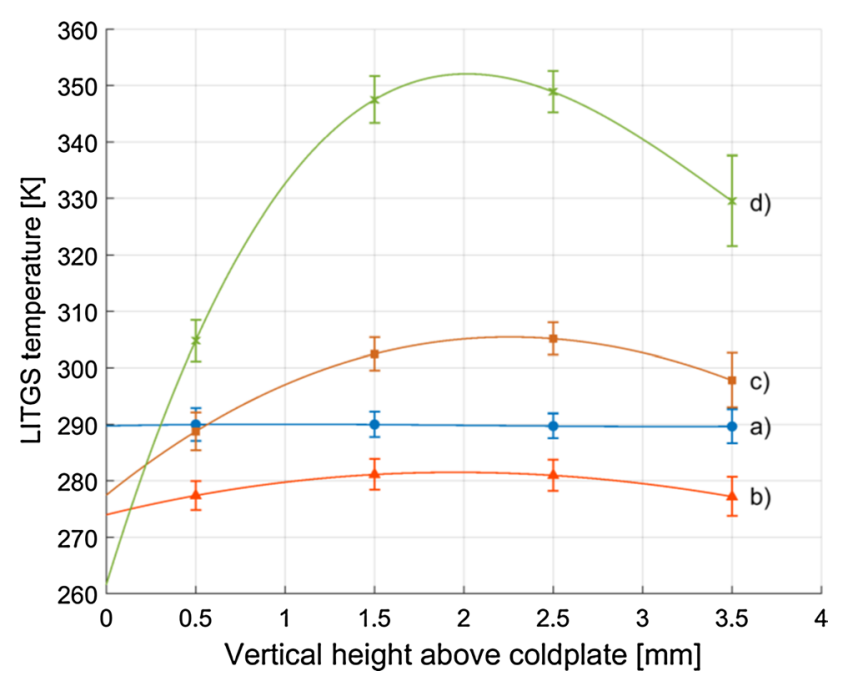

Fig. 6 Simultaneous measurements in a heated flow along a vertical line above a horizontal cooled plate. Data sets are shown for different temperatures, $T_{\mathrm{F}}$, of the flow as measured by a thermocouple at the nozzle exit. a Plate and flow at ambient temperature, $290 \mathrm{~K}$, with data adjusted by calibration. b Plate cooled and flow at ambient, $\mathbf{c}$ plate cooled, flow at $309 \mathrm{~K}$ and $\mathbf{d}$ plate cooled, flow at $341 \mathrm{~K}$ as indicated by a thermocouple at the nozzle. The curves are fitted splines as a guide to the eye 
the surface, at $3.5 \mathrm{~mm}$, is also slightly lower in temperature, presumably owing to cooling by entrained air at room temperature. A similar but more exaggerated non-uniformity in the temperature is shown in the data for the highest temperature flow at $341 \mathrm{~K}$ again as measured by a thermocouple at the nozzle exit. In this case, the cooling by the plate is pronounced for the boundary layer and some cooling is also observed at the top edge of the flow owing to the effects of cooler room air mixing with the flow. The temperatures derived from the LITGS data are hotter, by about $8 \mathrm{~K}$, than indicated by the thermocouple measurement at the nozzle. This is most likely a result of underestimation by the thermocouple as a result of slight conductive cooling of the gas in contact with the thermocouple itself.

\subsection{Extension to additional measurement points}

Although only four points have been measured in the present work, the method could be extended to more points if more detector channels are available. The four-channel oscilloscope used to acquire the data here could be replaced by a simpler, multichannel DAQ. It is also possible to generate signals from additional points using the present system by delaying signals from additional image "points" in a, second, longer optical fibre and registering them on one of the same photodiodes. Delays of the order of $500 \mathrm{~ns}$ can be introduced by a $100-\mathrm{m}$ optical fibre. Further multiplication using this approach could, in principle, allow a 2-D matrix of points to be measured simultaneously providing sample temperature measurements across a plane. This extension would require pencil-shaped pump beams, rather than sheet-like beams, to generate the thermal grating which would be interrogated by a sheet-like probe beam with appropriate imaging of the intersection plane on the fibre-optic/detector array.

\section{Conclusion}

The simultaneous measurement of temperature using LITGS at four points along a line has been demonstrated in this work. This "demonstration of principle" shows that by using fibre-optic coupling of signals to separate photodiode detectors, the limitation of streak cameras, which cannot provide both the required rise time and sweep duration, has been overcome.

Single-shot measurements have been recorded with a precision of $0.7 \%$ at room temperature and atmospheric pressure as defined by the standard deviation on 200 sequential measurements in nominally stable flows. Measurements of flows above 1 bar will be expected to allow higher precision as the duration of the signals increases at higher pressure providing a more precise measurement of the oscillation frequency (Williams et al. 2014). The present work shows that time- and space-resolved measurements can be taken with high precision using relatively inexpensive apparatus. Temperature gradients have been resolved both in flows and in boundary layers for approximately laminar flow across planar surfaces. A spatial resolution of $1 \mathrm{~mm}$ was achieved using 1:1 imaging of the linear interaction region on the fibre-optic detector array. The system is therefore capable of resolving small temperature differences of $5 \mathrm{~K} \mathrm{~mm}^{-1}$. This resolution can be readily improved simply by magnification of the interaction region by the imaging optics onto the detector array, provided that signal strengths can be maintained with adequate signal-tonoise ratio.

For flows across surfaces, extrapolation of the spline fits to the data points allows an estimation of the temperature at the surface for comparison with the values registered by the thermocouples. The reasonable agreement between the thermocouple values and estimates from extrapolated LITGS values gives some validation of the calibration method used to account for compositional variations in the flows.

This work illustrates the potential of multi-point LITGS for the study of fluid dynamic problems. The flows used here were nominally laminar but were constructed for the purposes of demonstrating the feasibility of the technique. More carefully designed and constructed flow systems should allow measurements that could be used to validate computational fluid dynamic models of flows. Nonetheless, the data presented here do show qualitatively the effects of flow mixing and boundary layer behaviour.

The ability to measure simultaneously the time-resolved temperature at multiple points, either in a line or on a plane, has potential for applications in internal combustion engines, e.g. for studies of heat release or heat loss to cylinder walls, hypersonic studies in shock tubes or blowdown facilities, e.g. for studies of boundary layer issues or other transient phenomena involving temperature gradients. It should be noted, however, that in circumstances where turbulence leads to inhomogeneous mixtures in the measurement volumes, the concentration of species such as fuels having a value of $m / \gamma$ differing significantly from that of air should, if possible, be kept to around $1 \%$ or less in order to minimize errors arising from compositional variation. Higher concentrations, together with a calibration method, were used in the present work in order to demonstrate the principle of the technique more clearly.

Acknowledgements This work was funded in part by the Engineering and Physical Sciences Research Council (EPSRC), UK, under Grant Number EP/M009424/1. CW is grateful to EPSRC and BP for personal financial support. 
Open Access This article is distributed under the terms of the Creative Commons Attribution 4.0 International License (http://creativecommons.org/licenses/by/4.0/), which permits unrestricted use, distribution, and reproduction in any medium, provided you give appropriate credit to the original author(s) and the source, provide a link to the Creative Commons license, and indicate if changes were made.

\section{References}

Bohlin A, Kliewer CJ (2013) Communication: two-dimensional gasphase coherent anti-Stokes Raman spectroscopy (2D-CARS): simultaneous planar imaging and multiplex spectroscopy in a single laser shot. J Chem Phys 138(22):221101

Bohlin A, Kliewer CJ (2014) Diagnostic imaging in flames with instantaneous planar coherent Raman spectroscopy. J Phys Chem Lett 5(7):1243-1248

Bohlin A, Patterson BD, Kliewer CJ (2013) Communication: simplified two-beam rotational CARS signal generation demonstrated in 1D. J Chem Phys 138(8):081102

Cummings EB (1994) Laser-induced thermal acoustics: simple accurate gas measurements. Opt Lett 19:1361-1363

Dreier T, Ewart P (2002) Coherent techniques for measurements with intermediate concentrations. In: Kohse-Höinghaus K, Jeffries JB (eds) Applied combustion diagnostics. Taylor and Francis, New York

Eckbreth AC (1996) Laser diagnostics for combustion temperature and species. Gordon and Breach, Philadelphia

Eichler HJ, Günter P, Pohl DW (1986) Laser-induced dynamic gratings. Springer, Berlin

Ewart P, Willman C, Williams BAO, Williams J, Stone CR (2015) Internal combustion engines. In: Proc. I. Mech. E. conference on internal combustion engines, $p$ 111. ISBN: 978-0-9572374-6-9
Hemmerling B, Stampanoni-Panariello A (1993) Imaging of flames and cold flows in air by diffraction from a laser-induced grating. Appl Phys B Photophys Laser Chem 57:281-285

Heppenheimer TA (2006) Facing the heat barrier: a history of hypersonics, NASA History Series (NASA SP-2007-4232)

Kays WM, Crawford ME (1993) Convective heat and mass transfer, 3rd edn. New York, McGraw-Hill

Kiefer J, Ewart P (2011) Laser diagnostics and minor species detection in combustion using resonant four-wave mixing. Prog Energy Combust Sci 37(5):525-564

Kohse-Höinghaus K, Jeffries JB (eds) (2002) Applied combustion diagnostics. Taylor and Francis, New York

Latzel H, Dreizler A, Dreier T, Heinze J, Dillmann M, Stricker W, Lloyd GM, Ewart P (1998) Thermal grating and broadband degenerate four-wave mixing spectroscopy of $\mathrm{OH}$ in high-pressure flames. Applied Physics B 67:667

Lucht R, Dunn-Rankin D, Walter T, Dreier T et al (1991) SAE Technical paper 910722. doi:10.4271/910722

Paul PH, Farrow RL, Danehy PM (1995) Gas-phase thermal-grating contributions to four-wave mixing. JOSA B 12(3):384-392

Schulz C, Sick V (2005) Tracer-LIF diagnostics: quantitative measurement of fuel concentration, temperature and fuel/air ratio in practical combustion systems. Prog Energy Combust Sci 31:75-121

Stevens R, Ewart P (2006) Simultaneous single-shot measurement of temperature and pressure along a one-dimensional line by use of laser-induced thermal grating spectroscopy. Opt Lett 31:1055-1057

Williams B, Edwards M, Stone R, Williams J, Ewart P (2014) High precision in-cylinder gas thermometry using laser induced gratings: quantitative measurement of evaporative cooling with gasoline/alcohol blends in a GDI optical engine. Combust Flame 161:270-279 